ISSN 0001-6012/2020/62/4/187-195 Acta Médica Costarricense, @ 2020 Colegio de Médicos y Cirujanos de Costa Rica

\title{
Original
}

\section{Respuesta virológica sostenida en pacientes con hepatitis C crónica, tratados con antivirales de acción directa: un estudio retrospectivo en Costa Rica}

(Sustained virological response in patients with chronic hepatitis $\mathrm{C}$ treated with direct acting antivirals, a retrospective study from Costa Rica)

Wagner Enrique Ramírez-Quesada, ${ }^{1}$ Marianella Madrigal-Borloz, ${ }^{2}$ Ricardo Barahona-García, ${ }^{3}$ Francisco Hevia-Urrutia ${ }^{4}$

\section{Resumen}

Introducción: Costa Rica es un país con un sistema de salud pública que ha permitido detectar oportunamente a los pacientes con hepatitis $\mathrm{C}$, y ofrecer un tratamiento con base en antivirales de acción directa (AAD) de última generación. No obstante, no se han publicado estudios que evalúen la respuesta de la población costarricense a estos fármacos.

Objetivo: describir la efectividad clínica del tratamiento con AAD en una cohorte tratada en la Caja Costarricense de Seguro Social (CCSS).

Afiliación de autores: 'Universidad de Costa Rica. 2Servicio de Gastroenterología, Hospital México, Caja Costarricense de Seguro Social. ${ }^{3}$ Servicio de Gastroenterología, Hospital "Dr. Calderón Guardia", Caja Costarricense de Seguro Social "Servicio de Gastroenterología, Hospital San Juan de Dios, Caja Costarricense de Seguro Social.

Trabajo realizado en: Hospital San Juan de Dios, Hospital "Dr. Rafael Ángel Calderón Guardia" y Hospital México, Caja Costarricense de Seguro Social.

Abreviaturas: $A A D$, antivirales de acción directa; $\mathrm{CHC}$, carcinoma hepatocelular; GLE, glecaprevir; VHB, hepatitis $B$; VHC, hepatitis C; PegIFN, inteferón pegilado; LDV, ledipasvir; O / / R / D, ombitasvir / paritaprevir / ritonavir / dasabuvir; PIB, pibrentasvir; RBV, ribavirina; RVS, respuesta virológica sostenida; SOF, sofosbuvir; VAR, variantes asociadas a resistencia; VEL, velpatasvir; VIH, virus de inmunodeficiencia humana; VOX, voxilaprevir.

凶wagner.ramirezquesada@gmail.com
Materiales y métodos: estudio retrospectivo de los expedientes clínicos de los pacientes tratados con sofosbuvir / ledipasvir, sofosbuvir / velpatasvir y ombitasvir / paritaprevir / ritonavir / dasabuvir, en tres hospitales nacionales para adultos de la CCSS, en 2017 - 2018. Se recolectaron variables epidemiológicas, clínicas y analíticas, y se compararon los resultados pre y postintervención.

Resultados: se reclutaron 139 pacientes; 22 fueron excluidos porque no cumplían los criterios. El análisis se realizó con 117 pacientes, de los cuales 101 tenían viremia documentada para determinación de la respuesta virológica sostenida (RVS). La mayoría de los pacientes fue costarricense, nacida en 1945 - 1965, con factores de riesgo para hepatitis C no documentados, sin cirrosis e infectada por el genotipo 1b. La RVS general de la población estudiada fue del 98 $\%$, sin notarse diferencia significativa entre pacientes cirróticos (94\%) y no cirróticos (100\%). Hubo una reducción significativa $(p<0,01)$ en: el índice de aspartato-aminotransferasa: número de plaquetas (APRI), el puntaje del Modelo para Enfermedad Hepática Terminal (MELD), la alaninoaminotransferase (ALT) y la bilirrubina total, para los pacientes tratados con AAD.

Conclusión: los antivirales de acción directa fueron efectivos en la población tratada en Costa Rica, con respuesta viral sostenida similar a aquella reportada en otros ensayos de vida real.

Descriptores: hepatitis C, antivirales, Costa Rica, respuesta virológica sostenida.

\section{Abstract}

Introduction: Costa Rica has a public healthcare system that made possible the detection of hepatitis $\mathrm{C}$ (HCV) infected patients and offer them treatment with last-generation direct-acting antivirals (DAA). Nonetheless, there has not been any published studies that evaluate the response of the Costa Rican population to these drugs. 
Aim: To describe the clinical effectiveness of direct acting antiviral treatment in a cohort treated in the Social Security Care from Costa Rica (CCSS).

Materials and Methods: Retrospective review of clinical records of all patients who were treated with: sofosbuvir/ledipasvir, sofosbuvir/velpatasvir and ombitasvir/paritaprevir/ritonavir/dasabuvir in three national adult hospitals from between 2017-2018. Epidemiological, clinical and laboratory data were collected, and pre- and post- treatment results were compared.

Results: 139 patients were recruited, 22 were excluded because they did not fulfill the inclusion criteria. The analysis was made with 117 patients; from which 101 had their viremia documented in their records for the determination of sustained virological response (SVR). The majority of patients were Costa Ricans born between 1945-1965, whose risk factors for hepatitis C were not documented, with a non-cirrhotic, genotype $1 \mathrm{~b}$ infection. Overall SVR was $98 \%$. There was not a significant difference of response between cirrhotic (94\%) and non-cirrhotic population (100\%). There was a significant reduction $(\mathrm{p}<0,01)$ in: Aspartate Aminotranferase to Platelet Ratio Index (APRI), the score of the Model for End-Stage Liver Disease (MELD), Alanine Aminotransferase (ALT) and total bilirubin in patients treated with DAA.

Conclusion: The direct acting antivirals were effective in population treated in our country, with SVR similar to those reported in real life studies from other regions of the world.

Keywords: hepatitis C, antivirales, Costa Rica, sustained viral virologic response.

Fecha recibido: 05 de febrero 2020

Fecha aprobado: 15 de octubre 2020

En 2017, el Reporte Global de Hepatitis de la Organización Mundial de la Salud (OMS) señaló que luego de la segunda mitad del siglo XX, ha habido una reducción en la incidencia mundial de hepatitis $\mathrm{C}(\mathrm{VHC})$. Los principales factores asociados a este hecho son la inversión en la pirámide poblacional, el declive de la generación "baby boomer" y la implementación de programas de salud pública, como por ejemplo el tamizaje activo y la prevención del intercambio de agujas para usuarios de drogas endovenosas (EV). ${ }^{1}$ Pese a esta tendencia epidemiológica, la incidencia global es perpetuada por prácticas de salud inseguras, el abuso de drogas EV y el comportamiento sexual riesgoso. ${ }^{2,3}$ La OMS clasifica al VHC como un problema de salud pública porque otras infecciones crónicas como el virus de inmunodeficiencia humana (VIH), tuberculosis o malaria, han sido blanco de distintas intervenciones poblacionales que han disminuido su mortalidad, pero en cambio la mortalidad relacionada con las hepatitis virales ha incrementado hasta alcanzar 1,34 millones de muertes en 2015, comportamiento que representa un incremento del $22 \%$, en 5 años. ${ }^{1}$ Los factores de riesgo para la infección por VHC de acuerdo con las guías de la Asociación Americana para Estudios de Enfermedades Hepáticas y la Asociación Europea para Estudios del Hígado, son varios, sin embargo, entre los más relevantes se encuentran: un comportamiento sexual de riesgo (múltiples parejas sexuales, hombres que tienen sexo con hombres, sexo receptivo o sin protección), transfusión de hemoderivados antes de 1992, uso de drogas EV o presencia de tatuajes.,3

Previo a la llegada de los antivirales de acción directa (AAD), el tratamiento estándar consistía en interferón pegilado (PegIFN) y ribavirina (RBV); 4 este régimen mostró incrementar las tasas de respuesta virológica sostenida (RVS), definida como la aviremia luego de 24 semanas de completar la terapia. ${ }^{4} \mathrm{La}$ RVS se convirtió en el equivalente de la curación virológica y se ha asociado con mejor calidad de vida, disminución en la progresión de la hepatopatía crónica y, en aquellos pacientes con fibrosis avanzada y cirrosis, la mitigación en las descompensaciones y la disminución del riesgo de carcinoma hepatocelular $(\mathrm{CHC})$, aunque sin anularlo del todo. ${ }^{3}$ Aun así, la terapia basada en IFN se asociaba con varios efectos adversos y los regímenes podían prolongarse por 48 semanas y, en algunos casos, hasta 72 semanas. Esto causaba un seguimiento complejo para el paciente y el médico, con RVS dependientes del genotipo, que en el mejor de los escenarios alcanzaba el $80 \%$ para GT 2 o 3 y tan bajas como 19,7 \% para el GT1. ${ }^{5}$ Dicha realidad incentivó investigaciones arduas y formulación de nuevas drogas con un mejor perfil de seguridad, que culminó con el desarrollo de los AAD que incluyen los inhibidores de proteasas, los inhibidores de polimerasa o NS5B y los inhibidores de NS5A., ${ }^{4}$

Los inhibidores de proteasa actúan en el complejo NS3 / NS4A, el cual tiene función dual de proteasa y helicasa y es relevante para la replicación viral. Los primeros dos compuestos en comercializarse fueron boceprevir y telaprevir, ambos forzosamente debían asociarse con a PegIFN / RBV y, por tanto, presentaban los mismos problemas que la terapia dual: especificidad genotípica estrecha y pobre barrera de resistencia genética. ${ }^{4}$

Los inhibidores de NS5B actúan en la proteína que sintetiza nuevo material genético y se clasifican como inhibidores nucleótidos y no-nucleótidos. Estos últimos, como dasabuvir, tienen un pobre perfil de resistencia y se usan principalmente como drogas adyuvantes en regímenes combinados. Por otro lado, los inhibidores nucleótidos cuentan con actividad pangenotípica y alta resistencia genética, lo que los hace ideales 


\section{Respuesta virológica hepatitis C en Costa Rica / Ramírez-Quesada et al}

para combinaciones, como en el caso del sofosbuvir (SOF), pero su principal debilidad es su potencial toxicidad. ${ }^{4}$

Por último, los inhibidores de NS5A se consideran como los AAD más potentes por su habilidad para inhibir la red lipídica necesaria para la replicación viral. Esto los ha convertido en gran elección para formulaciones combinadas por sus buenos perfiles de seguridad y eficacia. ${ }^{4}$

Estas nuevas drogas se han utilizado en diversos escenarios, como pacientes previamente tratados, ${ }^{7,8}$ población cirrótica, ${ }^{9} \mathrm{O}$ coinfección por hepatitis B (VHB) o virus de inmunodeficiencia humana (VIH). ${ }^{10}$

Tanto la Asociación Americana para Estudios de Enfermedades Hepáticas (AASLD de sus siglas en inglés) como la Asociación Europea para Estudio del Hígado (EASL por sus siglas en inglés), sugieren que todo paciente no expuesto a tratamiento, con infección activa y sin ninguna contraindicación, debe ser tratado., ${ }^{2,3}$ Se hace énfasis en los pacientes con fibrosis avanzada o cirrosis, manifestaciones extrahepáticas, recurrencia posttrasplante hepático $(\mathrm{TH})$ y cualquier condición que predisponga a una progresión acelerada, así como aquellos sujetos con alto riesgo de transmisión viral. ${ }^{2,3}$ Existen pocas contraindicaciones para el uso de los AAD, por ejemplo la combinación con inductores fuertes del CYP450 (como la carbamazepina). Los inhibidores de proteasa no deben utilizarse en cirrosis descompensada, ya que conducen a un incremento en la toxicidad. Por último, el SOF puede acumularse si el aclaramiento endógeno de creatinina es $<30 \mathrm{ml} / \mathrm{min} / 1,73 \mathrm{~m}^{2} .2,3$

La prevalencia real de VHC en nuestro país todavía no se ha determinado con certeza. De acuerdo con la bibliografía revisada, un estudio conducido en 2013 determinó la seroprevalencia de VHC, VHB y VIH, en una muestra de 100 indigentes de la zona metropolitana de San José. Este estudio reportó solo un 4 \% de seroprevalencia para VHC. Sin embargo, el diseño metodológico empleado impide inferir la prevalencia real en la población general. ${ }^{11}$ En 2012, Hevia publicó las características de los pacientes tratados con PegIFN / RBV, en una pequeña muestra. ${ }^{12}$

En Costa Rica, los AAD llegaron a estar disponibles hasta 2016 - 2017. Debido a la ausencia de estudios que evalúen las características de la población tratada con $\mathrm{AAD}$, se considera este trabajo de vital importancia para el país. Por tanto, se diseñó un estudio que permitiera describir la RVS en pacientes tratados con AAD, para adultos, durante 2017 - 2018.

\section{Métodos}

Se identificó a todos los pacientes con VHC crónica tratados con cualquiera de los siguientes regímenes de AAD: sofosbuvir / ledipasvir (SOF/LDV), sofosbuvir / velpatasvir (SOF / VEL), ombitasvir, paritaprevir, ritonavir y dasabuvir (O / P / R / D), en los tres hospitales nacionales de la Caja Costarricense de Seguro Social (CCSS), durante 2017 y 2018. Los pacientes excluidos fueron aquellos sin disponibilidad de expediente médico y con ausencia del reporte de carga viral, una vez finalizado el tratamiento. Los pacientes que descontinuaron el tratamiento durante el seguimiento también fueron excluidos.

La información demográfica (edad, país de origen, residencia, sexo), las manifestaciones clínicas y los estudios analíticos fueron extraídos del expediente clínico. Se recolectó la presencia de hepatopatía crónica o $\mathrm{CHC}$, la coinfección con VHB o VIH, las concentraciones de alanino aminotransferasa (ALT), las concentraciones de bilirrubina total, el conteo plaquetario, el puntaje MELD y la clasificación Child-Pugh. Además, se recolectó información radiológica procedente del ultrasonido de abdomen, así como los hallazgos endoscópicos. Se incluyeron además las manifestaciones extrahepáticas y los factores de riesgo para infección por VHC, definidos por las guías europea y norteamericana. ${ }^{2,3}$ Las variables asociadas al tratamiento fueron: el tipo de esquema, la exposición a terapia previa y la presencia de RVS.

Los pacientes se distribuyeron según la presencia de hepatopatía crónica, o de acuerdo con la evidencia de las siguientes características: hallazgos ultrasonográficos sugestivos de cambios crónicos en el parénquima hepático o hipertensión portal según la presencia de várices esofagogástricas (lo cual ha sido validado y comparado con otras modalidades de imágenes más específicas para el diagnóstico de hipertensión portal, como la elastografía de transición, resonancia magnética o tomografía). ${ }^{20-24}$ Se recabó además el índice de ALT: plaquetas (APRI) $>1$, lo cual ha sido validado como diagnóstico no invasivo para fibrosis en el contexto de VHC. ${ }^{13}$

La comparación entre variables categóricas fue realizada mediante $\mathrm{X}^{2}$, en tanto que las variables continuas se compararon con T-Student, una vez comprobada la distribución normal de los valores. Un valor $p<0,05$ fue considerado como estadísticamente significativo. Se empleó el programa SPPS para Windows.

Este estudio se apegó a la normativa internacional y nacional para la investigación en seres humanos. El protocolo de investigación fue aprobado por el Comité Ético Científico del Centro de Desarrollo Estratégico e Información en Salud y Seguridad (CENDEISSS) (Protocolo R018-SABI-00201).

\section{Resultados}

\section{Características generales}

139 pacientes fueron identificados durante el período en estudio. Tres pacientes fueron excluidos porque fallecieron al inicio del tratamiento y no recibieron la dosis programada, 14 sujetos carecían de la información necesaria acerca del uso de $\mathrm{AAD}$ en sus expedientes médicos. Hubo 4 pacientes que no finalizaron el tratamiento, según los registros de farmacia, y un paciente tuvo pérdida durante el seguimiento. Por lo tanto, se estudió un total de 117 pacientes.

La edad media fue de 59 años, con un rango de 21 a 89 años; el grupo de edad entre 50 y 59 años fue el más prevalente. 
El Cuadro 1 resume las principales características de los pacientes incluidos; un 79,5\% (n=93) de los pacientes era costarricense, con solo 8 originarios de EEUU y 4 de Nicaragua; el resto correspondía a nacionalidades variadas, como cubana, alemana, argentina, española o taiwanesa.
Como muestra el Cuadro 1, los factores de riesgo más frecuentes fueron las transfusiones y el uso de drogas EV, empero el 65,8 \% de la población estudiada no tenía ningún factor de riesgo identificable en el récord médico. Nueve pacientes presentaban coinfección VHC / VIH y dos, VHC /

\begin{tabular}{|c|c|}
\hline Parámetro & Pacientes $n=117(\%)$ \\
\hline Edad (promedio y rango) & $59,9(22-81)$ \\
\hline $\operatorname{Sex}(M / F)$ & $62 / 55$ \\
\hline \multicolumn{2}{|l|}{ Genotipo } \\
\hline No identificable & $5(4,3 \%)$ \\
\hline 1 (sin subtipo) & $5(4,3 \%)$ \\
\hline $1 \mathrm{a}$ & $28(23,9 \%)$ \\
\hline $1 b$ & $61(52,1 \%)$ \\
\hline 2 & $7(6 \%)$ \\
\hline 3 & $6(5,1 \%)$ \\
\hline 4 & $5(4,3 \%)$ \\
\hline \multicolumn{2}{|l|}{ Detalles del tratamiento } \\
\hline Sofosbuvir + ledipasvir & $46(39,3 \%)$ \\
\hline Sofosbuvir + ledipasvir + ribavirina & $15(12 \%)$ \\
\hline Ombitasvir + ritonavir + dasabuvir + paritaprevir & $11(9,4 \%)$ \\
\hline Sofosbuvir + velpatasvir & $48(41 \%)$ \\
\hline \multicolumn{2}{|l|}{ Duración del tratamiento } \\
\hline 12 semanas & $108(92 \%)$ \\
\hline 24 semanas & $9(8 \%)$ \\
\hline Respuesta viral sostenida documentada $(n=101)$ & $98(97,3 \%)$ \\
\hline \multicolumn{2}{|l|}{ Hepatopatía crónica (HC) } \\
\hline Sin hepatopatía crónica & $69(59 \%)$ \\
\hline HC No clasificable & $2(1,7 \%)$ \\
\hline Child Pugh A & $31(64,6 \%)$ \\
\hline Child Pugh B & $14(29,2 \%)$ \\
\hline Child Pugh C & $1(2,1 \%)$ \\
\hline \multicolumn{2}{|l|}{ Tratamiento previo } \\
\hline Sin exposición a tratamiento & $69(59 \%)$ \\
\hline Interferón pegilado alfa + ribavirina & $43(36,7 \%)$ \\
\hline Interferón pegilado alfa + ribavirina + telaprevir & 3 \\
\hline Interferón pegilado alfa + ribavirina + boceprevir & 3 \\
\hline Sofosbuvir + simeprevir & 2 \\
\hline Ledipasvir + sofosbuvir & 2 \\
\hline Ombitasvir + ritonavir + dasabuvir + paritaprevir & 3 \\
\hline Interferón pegilado alfa + ribavirina + simeprevir & 1 \\
\hline
\end{tabular}


VHB; todos estaban siendo tratados para sus otras infecciones virales. Un 59 \% ( $\mathrm{n}=69)$ de los pacientes no presentaba $\mathrm{HC}$, y de aquellos pacientes que cumplían los criterios establecidos, el 64,5\% ( $\mathrm{n}=31$ ) fue catalogado como ChildPugh A. Seis pacientes tenían un CHC documentado al inicio del tratamiento. Un 96,5\% de los pacientes ( $\mathrm{n}=113)$ no tenía manifestaciones extrahepáticas, pero 2 sujetos desarrollaron crioglobulinemia; otro paciente fue diagnosticado con eritema multiforme (EM) y el restante con porfiria cutánea tarda (PCT).

Desde una perspectiva virológica, los pacientes fueron agrupados según el genotipo (GT) del VHC para su análisis respectivo; 94 de ellos fueron GT 1, de los cuales el 52,1\% (n=61) presentaba el GT 1b, con el resto de genotipos con menor representación. Un total de 5 pacientes no presentaron documentación de su GT y ninguno de los pacientes manifestó infección por los GT 5 o 6. (Cuadro 1).

\section{Tratamiento empleado}

En total, 69 pacientes (59\%) no habían recibido tratamiento antiviral previo. El $41 \%$ ( $\mathrm{n}=47$ ) restante había recibido diversos esquemas terapéuticos, y 8 de ellos fueron expuestos a más de una combinación fCuadro 1). La estrategia básica de PegIFN / RBV fue el tratamiento utilizado con mayor frecuencia en estos pacientes.

Los regímenes de $\mathrm{AAD}$ empleados durante el período en estudio fueron: SOF / LDV, SOF / LDV / RBV, O / P / R / D y SOF / VEL. Este último fue usado en el $41 \%$ de los pacientes, seguido de SOF / LDV en un 39,3\% de los casos, y las otras 2 combinaciones con porcentajes menos representativos. Un 92,3 $\%$ de estos esquemas tuvieron 12 semanas de duración, y el restante, 24 semanas. Entre estos 8 pacientes con tratamiento prolongado, 4 correspondían a una clasificación Child-Pugh B, 2 de ellos tratados con SOF / LDV y 2 con SOF / VEL; los 3

\begin{tabular}{|c|c|c|c|c|}
\hline \multicolumn{5}{|c|}{$\begin{array}{l}\text { Cuadro 2. Respuesta virológica sostenida (RVS) a tratamiento, según presencia de } \\
\text { hepatopatía crónica (HC), genotipo y antiviral de acción directa (AAD) utilizado en los } \\
\text { pacientes infectados con hepatitis C crónica, tratados en los hospitales San Juan de } \\
\text { Dios, Rafael Ángel Calderón Guardia y México, en 2017-2018 }\end{array}$} \\
\hline HC y Child-Pugh & RVS n (\%) & Falla terapéutica & Respuesta \% & Valor p (IC: $95 \%$ \\
\hline $\mathrm{No} \mathrm{HC}$ & $59(59,6 \%)$ & 0 & $100 \%$ & $93,9-100$ \\
\hline No clasificable & $2(2 \%)$ & 0 & $100 \%$ & $0,28(15,8-100)$ \\
\hline A & $26(26,3 \%)$ & $1(50 \%)$ & $96 \%$ & $81-99,9$ \\
\hline B & $12(12,1 \%)$ & $1(50 \%)$ & $92 \%$ & $64-99,8$ \\
\hline C & 0 & 0 & No documentado & \\
\hline Total & 99 (100\%) & $2(100 \%)$ & $98 \%$ & $93,03-99,8$ \\
\hline \multicolumn{5}{|l|}{ Genotipo } \\
\hline No documentado & $4(4 \%)$ & 0 & $100 \%$ & \\
\hline 1 & $4(4 \%)$ & 0 & $100 \%$ & \\
\hline $1 a$ & $24(24,2 \%)$ & 0 & $100 \%$ & \\
\hline $1 b$ & $53(53,5 \%)$ & $2(100 \%)$ & $96 \%$ & \\
\hline 2 & $4(4 \%)$ & 0 & $100 \%$ & \\
\hline 3 & $6(6,1 \%)$ & 0 & $100 \%$ & \\
\hline 4 & $4(4 \%)$ & 0 & $100 \%$ & \\
\hline Total & 99 (100\%) & $2(100 \%)$ & $98 \%$ & \\
\hline \multicolumn{5}{|l|}{ Tipo de AAD utilizado } \\
\hline SOF / LDV* & $41(41,4 \%)$ & $1(50 \%)$ & $97,60 \%$ & $87-99,9$ \\
\hline SOF / LDV / RBV* * & $14(14,1 \%)$ & $1(50 \%)$ & $93,30 \%$ & $0,43(68-99,8)$ \\
\hline $\mathrm{O} / \mathrm{R} / \mathrm{D} / \mathrm{P} * * *$ & $5(5,1 \%)$ & 0 & $100 \%$ & $47,8-100$ \\
\hline SOF / VEL *** & $39(39,4 \%)$ & 0 & $100 \%$ & $90,9-100$ \\
\hline Total & 99 (100\%) & $2(100 \%)$ & $98 \%$ & $93,03-99,8$ \\
\hline
\end{tabular}


pacientes Child-Pugh A y el paciente no clasificado según ChildPugh, se trataron por 24 semanas utilizando SOF / VEL.

\section{Respuesta virológica sostenida}

El 100 \% de los pacientes sin HC que fueron tratados alcanzó una RVS. Este subgrupo representó el 59,6\% de la población tratada (Cuadro 2).

Entre los pacientes con cirrosis y un puntaje Child-Pugh A, un $96 \%$ alcanzó una RVS. Esta categoría de pacientes representó el 26,3 \% del total de individuos incluidos. En tanto, un 92 \% de los casos de pacientes con una escala Child-Pugh B, alcanzaron RVS.

Con la excepción del GT1b, el resto de pacientes infectados por otros GT alcanzaron RVS, mientras que este específicamente tuvo dos casos de falla terapéutica.

La relación entre la RVS obtenida de acuerdo con el AAD utilizado se detalla en el Cuadro 2. En general, la respuesta al tratamiento fue del 98 \% (CI 95 \%: 93,03 - 99,76). Ninguno de los tratamientos presentó diferencia estadísticamente significativa entre sí con respecto a la RVS $(p=0,43)$.

Los cambios entre inicio y final de tratamiento del APRI, MELD, bilirrubina total, ALT y conteo plaquetario, alcanzaron cambios estadísticamente significativos, como se observa en el Cuadro 3.

\section{Efectos secundarios de los AAD}

Tres pacientes debieron suspender su tratamiento por efectos adversos: una reacción cutánea con diarrea concomitante, una diarrea severa que desencadenó lesión renal aguda y un último paciente cuyo expediente no detalló la causa de la suspensión de los fármacos. Todos ellos fueron retratados con SOF / VEL, por 24 semanas. Los otros efectos adversos no mayores documentados fueron: cefalea, náuseas y una elevación mínima de la creatinina sérica basal.

\section{Discusión}

De acuerdo con nuestro conocimiento, el presente es el primer estudio que evalúa la efectividad clínica de los $\mathrm{AAD}$ en una cohorte de pacientes costarricenses con VHC. Los hallazgos demuestran una tasa de efectividad, medida por la RVS, cercana al $100 \%$, independientemente del tipo de AAD empleado.

En 10 referente a las características generales, es llamativo que un $65 \%$ de los pacientes no presentaba ninguna característica de riesgo en su expediente médico. Si bien el carácter retrospectivo del estudio puede sesgar este hallazgo, es imperativo que se diseñen mejores estrategias de salud pública para una evaluación oportuna de las poblaciones susceptibles a esta infección. ${ }^{2,3}$ Se recomienda que dicha estrategia se base en pruebas rápidas de laboratorio que permitan obtener resultados en minutos y detectar casos que ameriten evaluación exhaustiva. ${ }^{2}$ El análisis demográfico mostró que la mayoría de la población pertenece a la generación de "baby boomers", entre 55-75 años de edad Estudios epidemiológicos recientes han evidenciado que este grupo tiene una prevalencia 5 veces mayor de VHC crónica que la población general. ${ }^{2}$ Algunas cohortes de pacientes estadounidenses han señalado que los sujetos nacidos entre 1945 y 1965 representan el $75 \%$ de los pacientes infectados por VHC, lo cual concuerda con nuestros hallazgos, que identificaron a un $82 \%$ de la población en este rango de edad. Este hecho es relevante para la toma de decisiones en salud pública, ya que identifica un grupo poblacional de riesgo previamente ignorado en las políticas de tamizaje. Las sociedades médicas científicas internacionales recomiendan practicar tamizaje de cohortes por grupos etáreos, independientemente de la presencia de otros factores de riesgo clásicos. ${ }^{2,14,15 .}$

La prevalencia del GT 1 documentada en este estudio ( 80 \%) es similar a la reportada por otros previos. ${ }^{11,12}$ En concreto, el GT 1b representa más de la mitad de los casos, similar a lo evidenciado a nivel global, donde el GT1 representa el $46 \%$, y el $1 \mathrm{~b}$ un $22 \%$, seguido del 1a, 3, 2 y 4. . $^{16,17}$ La bibliografía mundial reporta que en Latinoamérica hay un gran subregistro acerca de

\begin{tabular}{|c|c|c|c|}
\hline \multicolumn{4}{|c|}{$\begin{array}{c}\text { Cuadro 3. Promedio de variables de laboratorio y escalas de fibrosis y } \\
\text { severidad de la población tratada con antivirales de acción directa, pre } \\
\text { y postintervención en los hospitales San Juan de Dios, Rafael Ángel } \\
\text { Calderón Guardia y México, en } 2017-2018\end{array}$} \\
\hline Parámetro (media) & Pretratamiento & Posttratamiento & Valor $p$ \\
\hline ALT IU / dL & 66,83 & 22,68 & $p<0,01$ \\
\hline Bilirrubina total mg / dL & 1,04 & 0,82 & $p<0,01$ \\
\hline Conteo plaquetario céls / $\mathrm{mm} 3$ & 165,04 & 151,29 & $p<0,01$ \\
\hline MELD* & 7,09 & 6,03 & $p<0,01$ \\
\hline APRI * * & 1,49 & 0,66 & $p<0,01$ \\
\hline
\end{tabular}




\section{Respuesta virológica hepatitis C en Costa Rica / Ramírez-Quesada et al}

la infección por VHC, con la mayoría de los datos provenientes de México, Perú, Argentina y Brasil, evidenciándose gran variabilidad en la prevalencia de los genotipos en la región. ${ }^{16,17}$ Si bien es cierto, las guías de tratamiento actuales sugieren que ante la disponibilidad de drogas con actividad pangenotípica la determinación del GT no es mandatoria, ${ }^{3}$ la identificación del genotipo más frecuente en el país en el período en estudio cobra relevancia epidemiológica. ${ }^{16}$

Antes del inicio del tratamiento con $\mathrm{AAD}$, la presencia de HC es bastante relevante, pues el plan terapéutico puede variar de acuerdo con este hallazgo. Los ensayos clínicos han mostrado una RVS > $90 \%$ con todos los AAD en pacientes sin cirrosis; ; 18,19 a la vez, los pacientes con hepatopatía crónica alcanzan una RVS similar en los análisis de subgrupos o en los ensayos diseñados específicamente para esta subpoblación. ${ }^{20}$ Aunque los resultados posttratamiento son esperables de acuerdo con la bibliografía publicada, una de las debilidades de este estudio reside en el proceso de definir hepatopatía crónica en los expedientes médicos, pues no había suficientes estudios diagnósticos como biopsia hepática o elastografía de transición (ET). Por tanto, las características endoscópicas, radiológicas y séricas fueron utilizadas como marcadores subrogados de hepatopatía. Mediante dicha definición operativa, los hallazgos correlacionan apropiadamente con la RVS alcanzada en otros estudios clínicos. ${ }^{21-23,13,24,20,25}$

La exposición a tratamiento previo desempeña también un papel importante cuando se decide cuál AAD utilizar, pero en nuestra cohorte, PegIFN+RBV fue el esquema más empleado. La evidencia actual sugiere que exposición previa a esta terapia no altera la decisión sobre cuál AAD prescribiri, ${ }^{2,3}$ En el caso de los pacientes ya expuestos a AAD, 3 de ellos fueron expuestos a O / R / D / P y el resto a SOF / LDV. Los estudios SAPPHIRE-I y II, PEARL-III y IV y TUROUIOSE-II, mostraron la seguridad de esta combinación en más de 2000 individuos con escasos efectos adversos, entre de los cuales destacan la diarrea y las reacciones mucocutáneas leves..$^{19,26-28}$ En el contexto de los tres regímenes suspendidos, el primer caso fue expuesto a múltiples fármacos por complicaciones intrahospitalarias, como infecciones nosocomialies y síndrome coronario agudo, así que afirmar causalidad asociada al AAD es debatible. En el segundo caso, la decisión de suspender el AAD fue derivada de la edad avanzada del paciente y por disponibilidad de otra combinación terapéutica; en el último escenario no se documentó en su expediente la causa aparente para suspensión. Ambos pacientes previamente expuestos a SOF / LDV fueron GT4, uno de ellos VIH positivo y únicamente uno con HC compensada. Esta combinación ha sido validada por guías internacionales con estudios pivote que muestran una RVS del $100 \%{ }^{2,3,29}$ Los otros efectos adversos documentados han sido claramente descritos en los ensayos ION, en los cuales náusea y cefalea estuvieron presentes en un pequeño porcentaje de los pacientes expuestos a SOF / LDV. ${ }^{10,18,30}$ La elevación de creatinina sérica es preocupante en el contexto de pacientes con disminución de la tasa de filtración glomerular por riesgo a la acumulación de metabolitos de SOF, lo cual puede producir necrosis tubular aguda y nefritis intersticial aguda; no obstante, estos pacientes, como lo fue en nuestro caso, tienden a recuperar su función renal luego de la suspensión farmacológica. ${ }^{30}$
La decisión de retratamiento luego de exposición a AAD de nueva generación es un punto por discutir, pues existe el riesgo teórico de desarrollar variantes asociadas resistencia (VAR) a inhibidores de proteasa o de NS5A. El análisis de estas puede considerarse útil para decidir un nuevo régimen para el paciente. ${ }^{31}$ Pese a esto, la detección de VAR no se encuentra disponible de forma general, y por tanto muchas combinaciones farmacológicas tienen evidencia para ser utilizadas como retratamiento; en el caso de SOF / VEL, la cual fue el tratamiento de elección, un estudio fase 2 demostró que esta combinación con RBV alcanza RVS de hasta el 97 \%.32 Aunque la RBV no fue utilizada, la RVS se alcanzó en 3 casos y el resto de pacientes se encontraban durante tratamiento al final del período en estudio. Otras opciones terapéuticas aprobadas para estos escenarios son $\mathrm{SOF} / \mathrm{VEL} / \mathrm{VOX}^{2,3,33}$ y GLE / PIB. . $, 3,34,35^{2}$

La terapia con AAD puede influenciar diversas variables de laboratorio y escalas para establecer la severidad de la HC. Un estudio prospectivo mostró que la mejoría del APRI, conteo plaquetario y elastografía de transición como marcadores de fibrosis hepática e hipertensión portal, puede ser alcanzada luego de la RVS con ayuda de AAD, así como una disminución concomitante del MELD. ${ }^{36} \mathrm{El}$ valor promedio de APRI obtenido fue altamente sugestivo de fibrosis $(>1)$ y luego del tratamiento hubo una mejoría significativa a $<1$; el conteo plaquetario fue menor al límite inferior normal y luego del tratamiento hubo una disminución significativa de 15000 plaquetas / $\mathrm{mm}^{3}$. Si bien es cierto que con la regresión de la fibrosis y caída concomitante de la presión portal debería haber una mejoría en el conteo, ${ }^{36,37}$ estudios anecdóticos han descrito efectos adversos hematológicos en $<1 \%$ de los casos. ${ }^{38}$

En el caso del MELD, ensayos como el ASTRAL-4 presentaron una mejoría de este luego de la RVS por AAD; ${ }^{20}$ en nuestra serie se logró alcanzar una disminución significativa de 1,07 puntos. Una caída en este puntaje sugeriría una mejor sobrevida en estos individuos; sin embargo, este fenómeno conocido como "purgatorio del MELD“, es una tema controversial, pues los pacientes con HC descompensada y valores más altos de MELD, no necesariamente desarrollarán una mejoría significativa luego de la RVS y podrían ser sacados de lista de espera de trasplante, cuando aún mantienen riesgo de desarrollar falla hepática aguda sobre crónica o $\mathrm{CHC}$, que pondría en riesgo sus vidas sin un trasplante hepático. ${ }^{39,40}$ Nuestra realidad nacional actual dicta que el período de espera para un trasplante no emergente es mayor a 6 meses, por lo que, siguiendo las guías internacionales, decidimos tratar a todos los pacientes.

Las manifestaciones extrahepáticas están claramente descritas en al bibliografía; la mayoría de estos fenómenos son de origen inmunológico, como la presencia de crioglobulinas, pero la amplia gama de estas podría afectar piel, riñones, cerebro y vías metabólicas. La crioglobulinemia mixta es la manifestación extrahepática más frecuente, aunque solo un $5-15 \%$ de los casos es clínicamente significativo, y puede manifestarse como una vasculitis de pequeño vaso secundaria a depósitos de crioglobulinas IgG e IgM.41,42 La porfiria cutánea tarda y el eritema multiforme son menos frecuentes, pero ha habido reportes acerca de su relación con VHC.. ${ }^{41,43}$ La RVS está asociada a la remisión de la crioglobulinemia en $>90 \%$ de los 
casos, secundaria a la mejoría en la homeostasis inmunológica y la porfiria cutánea tarda tiende a mejorar con el tratamiento con $\mathrm{AAD}$; los 4 pacientes con estas condiciones extrahepáticas presentaron remisión sintomática luego del tratamiento..41,42

Independientemente de su alta efectividad, los AAD no están exentos de falla terapéutica, determinada por la imposibilidad de alcanzar una RVS. Varias cohortes reportan tasas de falla entre 3,6 - 3,9 \% ${ }^{44,45}$ ante la presencia de diversos factores de riesgo como: HC descompensada, lo que afecta la farmacocinética de los AAD; infección por GT 3; coinfección VHC / VIH; presencia de VAR; dosificación errónea; mala adherencia; interacciones farmacológicas o prescripción de un régimen inapropiado. ${ }^{44} \mathrm{El}$ CHC tiene un papel interesante en la falla terapéutica, pues varios estudios han demostrado que incluso combinaciones de segunda generación podrían fallar en alcanzar al RVS. Se ha sugerido que esta neoplasia funciona como un santuario para el virus, o que el tumor induce cambios intravasculares hepáticos que alteran la farmacodinámica de los $\mathrm{AAD} .{ }^{46}$ Se documentaron únicamente 2 fallas terapéuticas, ambas con factores de riesgo (hepatopatía crónica, CHC o exposición a múltiples regímenes terapéuticos). Este tipo de casos son candidatos a combinaciones como VEL / SOF / VOX por 12 semanas o GLE / PIB, por 16 semanas. ${ }^{2}$

El estudio presenta ciertas limitantes por sus criterios de selección, ya que únicamente reclutó pacientes de la Seguridad Social. Además, pudo haber un sesgo de información por la ausencia de datos incompletos en el expediente médico. Otra limitante del estudio es la ausencia de datos a largo plazo, la cual impide conocer si existe alguna complicación tardía en los pacientes que alcanzaron RVS. Entre las fortalezas del estudio cabe mencionar que arrojó datos relevantes sobre la población infectada por VHC en el país, con una tasa inesperadamente alta de "baby boomers" portadores de la infección y con el GT 1b predominante, similar a otros países de América Latina.

En conclusión, este estudio retrospectivo de vida real revela que el uso de $\mathrm{AAD}$ en la población de pacientes con $\mathrm{VHC}$ alcanzó una RVS sobresaliente (>90 \%) para todos los esquemas utilizados.

Agradecimientos y colaboradores: los autores agradecen profundamente el tiempo brindado a todas las personas que tuvieron especial interés en la elaboración de este manuscrito, y a quienes facilitaron la recolección y análisis de datos.

\section{Referencias}

1. Kershenobich D, Torre-Delgadillo A, Aguilar-Valenzuela LM. Heading Toward the Elimination of Hepatitis C Virus. Rev Invest Clin. 2018;70:29.31.

2. AALSD-IDSA Guidance Panel. Hepatitis C guidance 2018 update: AASLDIDSA recommendations for testing, managing, and treating hepatitis $C$ virus infection. Clin Infec Dis. 2018; 67: 1477-1492.

3. Pawlotsky JM, Negro F, Aghemo A, Berenguer M, Dalgard O, Dusheiko G, et al. EASL Recommendations on Treatment of Hepatitis C 2018. J Hepatol. 2018; 69:461-511.

4. Jakobsen JC, Nielsen EE, Feinberg J, Katakam KK, Fobian K, Hauser G, et al. Direct-acting antivirals for chronic hepatitis C. Cochrane Database Syst. Rev. 2017; 9. DOI: 10.1002/14651858.CD012143.pub3.
5. Palumbo E. Pegylated interferon and ribavirin treatment for hepatitis $C$ virus infection. Ther Adv Chronic Dis. 2011; 2: 39-45

6. Götte M, Feld JJ. Direct-acting antiviral agents for hepatitis C: structural and mechanistic insights. Nat Rev Gastroenterol Hepatol. 2016;13:338-351.

7. Afdhal N, Reddy KR, Nelson DR, Lawitz E, Gordon SC, Schiff E, et al. Ledipasvir and Sofosbuvir for Previously Treated HCV Genotype 1 Infection. N Engl J Med. 2014;370:1483-93.

8. Werner CR, Schwarz JM, Egetemeyr DP, Beck R, Malek NP, Lauer UM, et al. Second-generation direct-acting-antiviral hepatitis $\mathrm{C}$ virus treatment: Efficacy, safety, and predictors of SVR12. World J Gastroenterol. 2016;22:8050.

9. Feld JJ, Jacobson IM. Hézode C, Asselah T, Ruane PJ, Gruener N. Sofosbuvir and Velpatasvir for HCV Genotype 1,2,4,5 and 6 Infection. N Engl J Med. 2015; 373:2599-2607.

10. Naggie S, Cooper C, Saag M, Workowski K, Ruane P, Towner WJ, et al. Ledipasvir and Sofosbuvir for HCV in Patients Coinfected with HIV-1. N Engl J Med.2015;373:705-13.

11. Leiva-Hidalgo J, Madrigal-Méndez A, Salas-Segura D. Seroprevalencia de hepatitis B, C y VIH en indigentes en Costa Rica. Rev. Costarric. Salud Pública. 2013;22:113-118.

12. Hevia F, Vargas J, Quirós C, Madrigal M, Barahona R, Rodríguez K, et al. Hepatitis C crónica en Costa Rica al 2012: Genotipos y respuesta al tratamiento con interferón pegilado (INF-PEG) y Ribavirina. ¿Qué hacer ahora?. Rev. Gastroenterol. Méx. 2012; 77 (Supl 2): 1-53.

13. European Association for the Study of the Liver, Asociación Latinoamericana para el Estudio del Hígado. EASL-ALEH Clinical Practice Guidelines: Noninvasive tests for evaluation of liver disease severity and prognosis. J. Hepatol.2015;63(1):237-264

14. Mahajan R, Liu SJ, Klevens RM, Holmberg SD. Indications for Testing Among Reported Cases of HCV Infection From Enhanced Hepatitis Surveillance Sites in the United States, 2004-2010. Am. J. Public Health. 2013;103(8):1445-9.

15. Smith BD, Morgan, RL, Beckett, GA, Falck-Ytter Y; Holtzman D, Teo CG, et al. Recommendations for the Identification of Chronic Hepatitis C Virus Infection Among Persons Born During 1945-1965. Morb. Mortl. Wkly. Rep. 2012; 61: $1-18$.

16. Gower E, Estes C, Blach S, Razavi-Shearer K, Razavi H. Global epidemiology and genotype distribution of the hepatitis $\mathrm{C}$ virus infection. J. Hepatol. 2014;61:45-57

17. Messina JP, Humphreys I, Flaxman A, Brown A, Cooke GS, Pybus OG, et al. Global distribution and prevalence of hepatitis $C$ virus genotypes. Hepatol. 2015; 61:77-87

18. Afdhal N, Zeuzem S, Kwo P, Chojkier M, Gitlin N, Puoti M, et al. Ledipasvir and Sofosbuvir for Untreated HCV Genotype 1 Infection. N Engl J Med. 2014;370:1889-1898.

19. Ferenci P, Bernstein D, Lalezari J, Cohen D, Luo Y, Cooper C, et al. ABT450/r-Ombitasvir and Dasabuvir with or without Ribavirin for HCV. N Engl J Med. 2014;370:1983-1992.

20. Curry MP, O’Leary JG, Bzowej N, Muir AJ, Korenblat KM, Fenkel JM, et al. Sofosbuvir and Velpatasvir for HCV in Patients with Decompensated Cirrhosis. N Engl J Med.2015;373:2618-28.

21. Yeom SK. Prediction of liver cirrhosis, using diagnostic imaging tools. World J. Hepatol. 2015; 7:2069.

22. Allan R. Accuracy of ultrasound to identify chronic liver disease. W World Gastroenterol. 2010;16:3510.

23. Gerstenmaier JF, Gibson RN. Ultrasound in chronic liver disease. Insights Imaging. 2014;5:441-55.

24. Pol S, Parlati L. Treatment of hepatitis C: the use of the new pangenotypic direct-acting antivirals in "special populations". Liver Int. 2018;38:28-33.

25. de Franchis R. Expanding consensus in portal hypertension. Report of Baveno VI Consensus Workshop: Stratifying risk and individualizing care for portal hypertension. J Hepatol. 2015; 63:743-52.

26. Feld JJ, Kowdley KV, Coakley E, Sigal S, Nelson DR, Crawford D, et al. Treatment of HCV with ABT-450/r-Ombitasvir and Dasabuvir with Ribavirin. N Engl J Med. 2014;370:1594-603. 


\section{Respuesta virológica hepatitis C en Costa Rica / Ramírez-Quesada et al}

27. Poordad F, Hezode C, Trinh R, Kowdley KV, Zeuzem S, Agarwal K, et al. ABT450/r-Ombitasvir and Dasabuvir with Ribavirin for Hepatitis C with Cirrhosis. N Engl J Med. 2014;370:1973-82.

28. Raedler LA. Viekira Pak (Ombitasvir, Paritaprevir, and Ritonavir Tablets; Dasabuvir Tablets): All-Oral Fixed Combination Approved for Genotype 1 Chronic Hepatitis C Infection. Am Health Drug Benefits. 2015;8:142-147.

29. Kohli A, Kapoor R, Sims Z, Nelson A, Sidharthan S, Lam B, et al. Ledipasvir and sofosbuvir for hepatitis $C$ genotype 4: a proof-of-concept, single-centre, openlabel phase 2a cohort study. Lancet Infect Dis. 2015;15:1049-54.

30. Brown PR, Sadiq O, Weick A, Lenhart A, Elbatta M, Fernandez C, et al. Acute Kidney Injury in Patients Undergoing Chronic Hepatitis C Virus Treatment With Ledipasvir/Sofosbuvir. Hepatol Commun. 2018;2:1172-8.

31. Wyles DL, Luetkemeyer AF. Understanding Hepatitis C Virus Drug Resistance: Clinical Implications for Current and Future Regimens. Top Antivir Med. 2017;25(3):7.

32. Gane EJ, Shiffman ML, Etzkorn K, Morelli G, Stedman CAM, Davis MN, et al. Sofosbuvir-velpatasvir with ribavirin for 24 weeks in hepatitis $C$ virus patients previously treated with a direct-acting antiviral regimen. Hepatol. 2017;66:1083-9.

33. Bourlière M, Gordon SC, Flamm SL, Cooper CL, Ramji A, Tong M, et al. Sofosbuvir, Velpatasvir, and Voxilaprevir for Previously Treated HCV Infection. N Engl J Med. 2017;376:2134-46.

34. Poordad F, Felizarta F, Asatryan A, Sulkowski MS, Reindollar RW, Landis CS, et al. Glecaprevir and pibrentasvir for 12 weeks for hepatitis $C$ virus genotype 1 infection and prior direct-acting antiviral treatment. Hepatol. 2017;66:389397.

35. Poordad F, Pol S, Asatryan A, Buti M, Shaw D, Hézode C, et al. MAGELLAN-1, Part 2: glecaprevir and pibrentasvir for 12 or 16 weeks in patients with chronic hepatitis $\mathrm{C}$ virus genotype 1 or 4 and prior direct-acting antiviral treatment failure. J Virus Erad. 2017;3 (Suppl 2): 13-36.

36. Knop V, Hoppe D, Welzel T, Vermehren J, Herrmann E, Vermehren A, et al. Regression of fibrosis and portal hypertension in HCV-associated cirrhosis and sustained virologic response after interferon-free antiviral therapy. J Viral Hepat. 2016;23:994-1002.

37. Kobayashi N, Iijima H, Tada T, Kumada T, Yoshida M, Aoki T, et al. Changes in liver stiffness and steatosis among patients with hepatitis $C$ virus infection who received direct-acting antiviral therapy and achieved sustained virological response. Eur J Gastroenterol Hepatol. 2018; 30:546-551.

38. Dahal S, Upadhyay S, Banjade R, Dhakal P, Khanal N, Bhatt VR Thrombocytopenia in patients with chronic hepatitis C virus infection. Mediterr J Hematol Infect Dis. 2017;9:2017-2019.

39. Terrault NA, Hassanein TI. Management of the patient with SVR. J Hepatol 2016;65:120-9.

40. Shah H, Bilodeau M, Burak KW, Cooper C, Klein M, Ramji A, et al. The management of chronic hepatitis C: 2018 guideline update from the Canadian Association for the Study of the Liver. Can Med. Assoc J. 2018;190:677-87.

41. Polo ML, Laufer N. Extrahepatic manifestations of HCV: the role of direct acting antivirals. Expert Rev Anti-Infect. Ther. 2017;15:737-46.

42. Degasperi E, Aghemo A, Colombo M. Treatment of Extrahepatic Manifestations of Hepatitis C Virus. Clin Liv Dis. 2017;21:631-43.

43. Cacoub P, Bourlière M, Lübbe J, Dupin N, Buggisch P, Dusheiko G, et al. Dermatological side effects of hepatitis $\mathrm{C}$ and its treatment: Patient management in the era of direct-acting antivirals. J Hepatol. 2012;56:455-63.

44. Salmon D, Trimoulet P, Gilbert C, Solas C, Lafourcade E, Chas J, et al. Factors associated with DAA virological treatment failure and resistance-associated substitutions description in HIV/HCV coinfected patients. World J Hepatol. 2018;10:856-66.

45. Kondili LA, Gaeta GB, Brunetto MR, Di Leo A, Iannone A, Santantonio TA, et al. Incidence of DAA failure and the clinical impact of retreatment in real-life patients treated in the advanced stage of liver disease: Interim evaluations from the PITER network. PLoS ONE. 2017;12:e0185728.

46. Prenner SB, VanWagner LB, Flamm SL, Salem R, Lewandowski RJ, Kulik L. Hepatocellular carcinoma decreases the chance of successful hepatitis $C$ virus therapy with direct-acting antivirals. J Hepatol. 2017;66:1173-81. 